\title{
Eating behaviour patterns in Chinese children aged 12-18 months and association with relative weight - factorial validation of the Children's Eating Behaviour Questionnaire
}

\author{
Ying-Ting Cao ${ }^{1,2}$, Viktoria Svensson ${ }^{1}$, Claude Marcus ${ }^{1}$, Jing Zhang $^{2}$, Jian-Duan Zhang ${ }^{2^{*}}$ and Tanja Sobko ${ }^{1 *}$
}

\begin{abstract}
Background: Eating behaviours have been suggested relating to obesity development. The Children's Eating Behaviour Questionnaire (CEBQ) is a parent-report measure constructed to assess multiple dimensions of eating behavior for children. This study aimed to test the validity of the Chinese version of Children's Eating Behaviour Questionnaire (CEBQ) in Chinese children aged 12-18 months. We examined factor structure and the reliability of the Chinese version of the CEBQ, the associations between children's eating behaviours and children's weight (BMI SDS) were assessed.

Methods: 219 questionnaires were filled out by the caregivers, approached in community health care centers in two cities in China. BMI of each child was calculated and converted to BMI SDS. Factor validation (Principal Component Analysis, exploratory factor analysis) on all CEBQ items was performed and gender difference in eating behaviours was examined. Correlations between eating behaviours and the child's BMI SDS were analyzed by linear regression analysis controlling for gender, parental combined weight, and education.

Results: The factor analysis revealed a seven-factor solution, with factor 'food responsiveness' (FR) split into two. 'Satiety responsiveness' (SR) and 'Enjoyment of food' (EF) factors were not detected. Interestingly, boys scored higher than girls in the FR scales, whereas girls had a higher score in 'food fussiness' (FF) scale.

Conclusions: We conclude that although a valuable psychometric instrument, CEBQ might be affected by age and cultural differences. Therefore, adjusting it in order to fit the Chinese population was suggested. We did not find an association between eating behaviours and children's BMI SDS, when it was controlled for gender and parental weight.
\end{abstract}

Keywords: Eating behaviour, CEBQ, Chinese overweight children, obesity, factorial validation

\section{Background}

Childhood obesity has become one of the most serious public health challenges worldwide in the last century, with 42 million overweight children under the age of five. The vast majority (80\%) of these live in low and middle-income countries [1]. This trend has affected

\footnotetext{
* Correspondence: jd_zh@mail.hust.edu.cn; Tanja.sobko@ki.se

'Karolinska Institutet, Department of Clinical Science, Intervention and Technology, Division of Pediatrics, Karolinska University Hospital Huddinge, SE 14186 Stockholm, Sweden

${ }^{2}$ Tongji Medical College School of Public Health, Huazhong University of Science and Technology, 13 Hangkong RD, Wuhan, 430030, P.R.China Full list of author information is available at the end of the article
}

China and the prevalence of childhood obesity in some urban Chinese populations is already reached similar levels of the developed countries [2]. In 2005, total $7.73 \%$ of Chinese youth were overweight and $3.71 \%$ of them were obese. Partly explained by the explosive economic development and improvement of living conditions in China [3], general raise in income in China today enables a decreasing consumption of cereals and starchy roots, and an increasing consumption of highcaloric foods [4]. In addition, fast food and snack consumption together with increased TV screen time has 
been suggested to have contributed to the increase of childhood obesity prevalence in China [5].

Different patterns of eating behaviours have been suggested to influence the weight gain. For example, Schachter [6] showed that responsiveness to satiety, low in obese individuals, leads to failure to regulate their energy intake and to overeating. Furthermore, speed of eating was indicated as important factor for adiposity development $[7,8]$.

A few standardized psychometric tools to measure children's eating behaviours have been developed. Some examples of these are the Dutch Eating Behaviour Questionnaire (DEBQ) [9], the Children's Eating Behaviour Inventory (CEBI) [10] and Children's Eating Behaviour Questionnaire (CEBQ) [11]. The latter is regarded as the most comprehensive instrument to assess children's eating behaviour and it has been validated with high reliability and validity in several countries [11-14] and recently even in Sweden [15]. It describes children's eating behaviour from eight dimensions. Satiety responsiveness (SR) assesses the sensitivity to the internal food cues or the threshold in each individual when deciding to stop eating. Overweight children are prone to eat more and fail to regulate their food intake, leading to overeating and obesity, compared with normal weight children [16]. The Enjoyment of food (EF) and Food responsiveness (FR) both assess general food preference in children as well as the desire to eat [12]. The former assesses the general appetite and the latter assesses the level of appetite and the intention of eating under external cues [12]. Drink desire (DD) is about preferring sweet drinks, mostly without thirst or hunger [17]. Slowness in eating (SE) and Food fussiness (FF) reflect low interest and enjoyment of food. Studies on speed of eating have shown that obese children complete a meal faster than the lean children $[7,8]$. Fussy eaters are inclined to eat less and slower [18-20] leading to a slower weight gain $[20,21]$. At the same time, regarding the relationship between fussy eating and weight, no difference was found between normal weight and obese children [20]. Emotional overeating (EOE) and Emotional under eating (EUE) refer to the increase or decrease in eating under negative emotions, involving stress [22]. According to 'psychosomatic theory' [23], EOE under negative emotion could lead to excessive weight gain.

CEBQ has been validated for both Dutch and Portuguese populations $[13,14]$. A similar Swedish study validated the CEBQ among a Swedish young population which belongs to a big project on obesity prevention and intervention focus on diet, sleep and physical activities in Sweden [24]. Part of this project is conducted in China, and we therefore intended to validate the CEBQ in a Chinese context.
The present study aims to examine the factor structure of the original CEBQ and investigates its applicability in Chinese culture background. We also hypothesized that eating behaviours is could be associated with young children's relative weight.

\section{Methods}

\section{Procedures and participants}

The CEBQ was translated into Chinese (Mandarin), read and corrected by several Chinese native speakers. The Chinese version was then translated back to English, thereafter adjusted and translated again to Chinese in order to ensure the congruence between the two different languages.

A total of 219 children with 114 boys and 105 girls, aged from 12 to 18 months from two Chinese cities (Zhenjiang and Wuhan) participated in this study. Children within this age range and without physical or psychological problems were recruited from three local community health centers in Zhenjiang and Wuhan respectively, after parents' understanding and signing the informed consent form. Children's weight and height were measured on-spot at the community health care centers, using a calibrated infant length and weight scale (WSH-I, Wuhan computer software development Co. Ltd.). At the same occasion, all 219 questionnaires were completed by 184 parents and 35 grandparents with the assistance of study investigators when necessary. Each questionnaire was filled out only by one parent or grandparent, the person who took care of the child in the daily life. The demographic and anthropometric characteristics of the sample, as well as parental educational level were assessed (Table 1). Chinese percentiles and SD curve for boys and girls aged from 0-18 years old [25] were adopted as cutoff of overweight and obesity for children. The threshold for parents was according to Chinese adult BMI reference [26] with 24 $\leq \mathrm{BMI}<28$ regarded as overweight, and $\mathrm{BMI} \geq 28$ regarded as obesity.

\section{Data processing}

The CEBQ consists of 35 items in the form of eight sub-scales, with each sub-scale loaded by $3-6$ items. The items are rated on a five-point Likert scales, from 1 - $5(1=$ never, $2=$ seldom, $3=$ sometimes, $4=$ often, 5 $=$ always).

Body mass index (BMI) was calculated for parents and children respectively; children's BMI percentile was calculated according to Chinese latest reference. BMI for the child was adjusted for age and gender according to an international standard, BMI SDS [27]. Parents were divided into two groups given their calculated BMI. One group contained no overweight or obese parents; the 
Table 1 Demographic and BMI characteristics of the sample $(n=219)$

\begin{tabular}{|c|c|c|}
\hline & Mean (SD), Range & $\mathrm{N}(\%)$ \\
\hline Children's BMI SDS & $0.66(1.15),(-3.7-5.3)$ & \\
\hline Children's weight categories(a) & BMI & \\
\hline Underweight $\left(<5^{\text {th }}\right)$ & 12.5 & $1(0.5)$ \\
\hline Normal weight $\left(5^{\text {th }} \leq \mathrm{BMI}<85^{\text {th }}\right)$ & 17.2 & $111(50.7)$ \\
\hline Overweight $\left(85^{\text {th }} \leq \mathrm{BMl}<95^{\text {th }}\right)$ & 18.6 & $48(21.9)$ \\
\hline Obese $\left(95^{\text {th }} \leq \mathrm{BMI}\right)$ & 20.5 & $59(26.9)$ \\
\hline \multicolumn{3}{|l|}{ Parental BMI $\left(\mathrm{kg} / \mathrm{m}^{2}\right)$} \\
\hline Mother & $21.1(2.6),(16.6-32.0)$ & \\
\hline Father & $23.0(2.9),(16.5-33.9)$ & \\
\hline \multicolumn{3}{|l|}{ Combined parental weight } \\
\hline No overweight or obese parent & $128(58)$ & \\
\hline At least one overweight or obese parent & $91(42)$ & \\
\hline Parental weight categories (b) & & Mother/Father \\
\hline Underweight (BMI < 18) & & $16 / 7(7.3 / 3.2)$ \\
\hline Normal weight $(18 \leq \mathrm{BMI}<24)$ & & 178/137 (81.2/62.6) \\
\hline Overweight $(24 \leq \mathrm{BMI}<28)$ & & 20/64 (9.1/29.2) \\
\hline Obese $(28 \leq \mathrm{BMI})$ & & $5 / 11(2.3 / 5)$ \\
\hline \multicolumn{3}{|l|}{ Parental education } \\
\hline University and college & & $145 / 159(66.2 / 72.6)$ \\
\hline High school & & $68 / 57(31.1 / 26)$ \\
\hline Elementary school & & $6 / 3(2 / 7 / 1.4)$ \\
\hline
\end{tabular}

a Chinese children's reference (Li H, Ji CY, Zong XN, \& Zhang YQ, 2009);

${ }^{b}$ Chinese adults' BMI criteria (Ji CY,2005);

other group contained at least one overweight or obese parent.

\section{Statistical procedures}

All statistical analyses were conducted in STATISTICA 9.0 (data analysis software system, version 10. StatSoft, Inc. http://www.statsoft.com). In order to understand the underlying structure of the Chinese translation and ascertain whether it is similar to the original scale, exploratory factor analysis (EFA) was performed by using Varimax rotation on all items of the CEBQ. In order to allow factor inter-correlated, we used SPSS program to run this oblique rotation (Oblimin direct). Since the original CEBQ had an eight-factor structure, the number of factors was equally set to eight. Threshold for factor loadings was set as 0.6. Factors' reliability was assessed by using Cronbach's alpha coefficient. 0.7 was considered as general Cronbach's alpha cutoff, but the value could be lower for research compared to clinical situation [28]. Apart from 0.6 we even tried loading with 0.4 for factor analysis.

Score differences in factors between boys and girls and parental combined weight groups were tested by the independent $\mathrm{t}$-test. The correlation between sub-scales of the CEBQ was analyzed by Pearson correlation.
Finally, linear regression was used to test the association between children's BMI SDS (dependent variable) and each eating behaviour scale, controlling for gender, parental combined weight and parental educational level. We additionally checked a simple correlation between child BMI SDS and the CEBQ scales in order to avoid the possible attenuating effect of parental weight and other confounding factors.

\section{Results}

\section{Structure of translated scale and reliability}

The factor analysis revealed a seven-factor solution (SE, EUE, FF, FR 1, DD, EOE, FR 2) with factor FR split into two (FR 1, FR 2). Variability among all 35 items was $52.1 \%$, in terms of the seven factors (Table 2 ). Items belonging to each factor in Table 2 were loaded above 0.6. However, we omitted one item (number 5) in factor 1 (see table 2 and appendices) even though it loaded larger than 0.6. Because this item originally belonged to factor EF (enjoyment of food), rather than the other three homogenous items (all belong to factor SE (slowness of eating)), so it was excluded in factor SE.

Factor structure was not affected by running Oblimin rotation in SPSS program compared with the results 
Table 2 Factor loadings on Varimax Rotated Solution of Principal Components analysis $(C E B Q, N=219)$

\begin{tabular}{|c|c|c|c|}
\hline Scale name and items & Loading & Scale name and items & Loading \\
\hline Slowness in eating (Factor 1: $11.7 \%$ variance) & & Food responsiveness 1 (Factor 4: 6.2\% variance) & \\
\hline 4. My child finishes his/her meal quickly & 0.66 & 28. Even if my child is full up s/he finds room to eat his/her & 0.67 \\
\hline 8. My child eats slowly & 0.76 & favourite food & \\
\hline 18. My child takes more than 30 minutes to finish a meal & 0.63 & $\begin{array}{l}\text { 34. If given the chance, my child would always have food in his/her } \\
\text { mouth }\end{array}$ & 0.68 \\
\hline \multicolumn{4}{|l|}{ Emotional under-eating (Factor 2: 6.0\% variance) } \\
\hline 11. My child eats less when $s /$ he is tired & 0.62 & Drink desire (Factor 5: 7.1\%variance) & \\
\hline 23. My child eats more when she is happy & 0.60 & 6. My child is always asking for a drink & 0.80 \\
\hline 25.My child eats less when upset & 0.76 & $\begin{array}{l}\text { 29. If given the chance, my child would drink continuously } \\
\text { throughout the day }\end{array}$ & 0.80 \\
\hline Food fussiness (Factor 3: 6.8\% variance) & & 31. If given the chance, my child would always be having a drink & 0.82 \\
\hline 7. My child refuses new food at first & 0.71 & Emotional overeating (Factor 6: 6.6\%variance) & \\
\hline 10. My child enjoys tasting new foods & 0.77 & 2. My child eats more when worried & 0.65 \\
\hline \multirow{5}{*}{$\begin{array}{l}\text { 32. My child is interested in tasting food s/he hasn't } \\
\text { tasted before }\end{array}$} & 0.75 & 13. My child eats more when annoyed & 0.74 \\
\hline & & 15. My child eats more when anxious & 0.77 \\
\hline & & Food responsiveness 2 (Factor 7: 7.7\% variance) & \\
\hline & & 12. My child is always asking for food & 0.65 \\
\hline & & 19. Given the choice, my child would eat most of the time & 0.64 \\
\hline
\end{tabular}

using Varimax rotation in STATISTICA program. It was neither affected by setting 0.4 as loading for factor analysis.

Internal consistency indicated by the Cronbach's alpha coefficient ranged from 0.52 to 0.80 for the CEBQ subscales (Table 3).

\section{Gender and combined parental weight difference}

Significant gender differences in both food responsiveness sub-scales (FR 1 and FR 2) were identified (Table 4); boys scored higher than girls on FR 1 (mean 2.42 (SD 0.83) versus 2.08 (SD 1.01), $\mathrm{p}=0.01$ ) and FR 2 (mean $3.34(0.83)$ versus $3.00(1.04), \mathrm{p}=0.01)$. When it comes to Food fussiness (FF), boys scored significantly lower than girls (2.30 (0.84) VS 2.57 (0.87), p = 0.02). No significant difference in factors was found by combined parental weight.

Correlations between sub-scales of CEBQ and associations between children's weight and their eating behaviours
Our study indicated positive inter-correlations in the four 'food-approach' scales (FR 1, DD, EOE, FR 2) and the two 'food-avoidant' scales (SE, FF). Significant negative correlations $(\mathrm{p}<0.05)$ were found between the two groups of scales (FF vs DD, r $=-0.16$, and FF vs FR 2, r $=-0.24$, , Sub-scales EOE and EUE $(r=0.21)$ were positively correlated $(\mathrm{p}<0.05)$, in accordance with the previous studies. The correlations between sub-scales of CEBQ are shown in Table 5.

There were no associations between child's BMI SDS and eating behaviours when controlled for gender, parental combined weight and parental educational level. We did not find any associations either between child's BMI SDS and eating behaviours (the factors revealed by factor analysis in this study), when we performed a simple correlation.

\section{Discussion}

To our knowledge, this is the first time the CEBQ is tested in China. Also, this study is the first attempt to

Table 3 Factor reliability of the CEBQ $(\mathbf{N}=219)$

\begin{tabular}{lll}
\hline & Cronbach's alpha & Average item-total correlation (range) \\
\hline Slowness in eating & 0.71 & $0.48(0.43-0.66)$ \\
Emotional undereating & 0.60 & $0.33(0.30-0.46)$ \\
Food fussiness & 0.71 & $0.53(0.49-0.57)$ \\
Food responsiveness 1* & 0.52 & $0.35(0.35-0.35)$ \\
Desire to drink & 0.80 & $0.65(0.59-0.68)$ \\
Emotional overeating & 0.69 & $0.50(0.44-0.55)$ \\
Food responsiveness 2** & 0.55 & $0.38(0,38-0.38)$ \\
\hline
\end{tabular}

* contains item 28 and $34 ;{ }^{* *}$ contains item 12 and 19 ; item numbers refer to table 1 
Table 4 Gender difference in eating behavious (subscales)

\begin{tabular}{lccc}
\hline Factors & Boy (N = 114) & Girl (N = 105) & P \\
\cline { 2 - 3 } & Mean (SD) & Mean (SD) & \\
\hline Slowness in eating & $2.90(0.65)$ & $2.96(0.61)$ & 0.43 \\
Emotional undereating & $3.09(0.90)$ & $2.95(0.87)$ & 0.23 \\
Food fussiness & $2.30(0.84)$ & $2.57(0.87)$ & $0.02^{*}$ \\
Food responsiveness 1 & $2.42(0.83)$ & $2.08(1.01)$ & $0.01^{*}$ \\
Desire to drink & $3.14(0.86)$ & $2.96(0.99)$ & 0.14 \\
Emotional overeating & $1.82(0.68)$ & $1.75(0.64)$ & 0.46 \\
Food responsiveness 2 & $3.34(0.83)$ & $3.00(1.04)$ & $0.01^{*}$ \\
\hline
\end{tabular}

FR $1=$ item 28, 34; FR 2 = item12, 19. ${ }^{*} p<0.05$

assess eating behaviour pattern among young Chinese children. We examined whether eating behaviours measured by the CEBQ are associated with children's weight in a sample of Chinese children at the age of 12-18 months.

Compared with the eight-factor structure in the original study [11], our study revealed seven CEBQ subscales (with two separate FR factors), and two factors could not be detected in our sample (EF and SR). Each sub-scale contained the items originally belonging to it, with an exception of the sub-scale factor SE, which in our study also had items originally belonging to another sub-scale (EF), such as item 5 (my child is interested in food).

Surprisingly, the original FR factor was split into two factors, each having two items loaded onto it, called FR 1 and FR 2 respectively. The items loaded onto FR 1 were 'Even if my child is full up, s/he finds room to eat his/her favourite food' and 'If given the chance, my child would always have food in his/her mouth'. The items loaded onto FR 2 were 'My child is always asking for food' and 'Given the choice, my child would eat most of the time'. However, one possible reason for two FRs could be the interpretation for 'always have food in the mouth'. Younger children are prone to keep the food in the mouth, and not swallowing immediately compared to older children.

Table 5 Correlations between CEBQ sub-scales

\begin{tabular}{|c|c|c|c|c|c|c|c|}
\hline & $\mathrm{SE}^{\mathrm{a}}$ & EUE $^{\mathbf{b}}$ & $\mathrm{FF}^{\mathrm{c}}$ & $\mathrm{FR}^{\mathrm{d}} 1$ & $\mathrm{DD}^{\mathrm{e}}$ & $\mathrm{EOE}^{f}$ & FR 2 \\
\hline SE & - & - & - & - & - & - & - \\
\hline$\overline{\text { EUE }}$ & 0.04 & - & - & - & - & - & - \\
\hline $\mathrm{FF}$ & $0.26^{*}$ & 0.05 & - & - & - & - & - \\
\hline FR 1 & 0.07 & -0.05 & -0.06 & - & - & - & - \\
\hline $\mathrm{DD}$ & -0.06 & 0.18 & $-0.16^{*}$ & $0.16^{*}$ & - & - & - \\
\hline$\overline{\mathrm{EOE}}$ & -0.05 & $0.21^{*}$ & 0.05 & $0.16^{*}$ & $0.21^{*}$ & - & - \\
\hline FR 2 & 0.02 & 0.10 & $-0.24^{*}$ & $0.24^{*}$ & $0.28^{*}$ & 0.13 & - \\
\hline
\end{tabular}

We only show 19 items in table 2, although the original CEBQ scale has 35 items. The other missing 16 items either did not load on any factors (loadings $<0.6$ ) or were excluded even though loadings $>0.6$ (item 5), or because they originally belonged to the two missing factors (EF and SR). The comparison of factor structure of this study with the original scale structure was indicated in additional file 1 . When we lowered the loading from 0.6 to 0.4 , it brought more items loaded on each factor. However, it didn't change the final factor results.

Regarding the two missing sub-scales, compared to the original factor structure of the CEBQ (EF and SR), they might not be applicable for the early age of the Chinese study group. The impact of the Chinese history and culture may also be taken into account of. The concept that 'a chubby baby is a healthy baby' is still presents today in spite of the growing awareness of childhood obesity in China. The one-child policy in China in the 1980s aggravated the situation when the only child became the cosset in the family. Moreover, in China, many grandparents take care of their only grandchild in an excessive way, traditionally reflecting in overfeeding practices. As a result, they provide their grandchildren with high energy-dense foods in far greater than needed amounts, increasing the potential risk for adiposity [29]. Consequently, young children could be tired of the food from an early age since they are always overfed, which could explain the missing EF and SR sub-scales.

Cronbach's alpha coefficient normally ranges between 0 and 1 but actually no lower limit to the coefficient is existed and the closer it is to 1 , the stronger consistency the items in the scale [30]. In our study, low scores of the two FRs could be due to the inapplicability among particularly young children.

An interesting finding of this study is a significant gender difference in the FF and the two FR factors, suggesting that boys might be more interested in food than girls and girls might be more 'picky' than boys at an early age. Contradictory gender results in picky eating have been reported so far: either no gender differences $[31,32]$ or boys scoring higher of food fussiness than girls [33]. However, another study has just recently reported the gender difference of 'food responsiveness' when using the Baby Eating Behaviour Questionnaire: male infants were slightly more responsive to food than female infants [34]. The issue is complicated by many aspects, for example, age. Addessi and others showed that this behaviour peaks at the age of $2-6$, decreasing later all the way through adolescence [35].

In our study sample we found an unusually high proportion of overweight and obesity being almost half of the sample size. Although this should be checked in a bigger population, we still would like to report this. As mentioned above, this could be due to a common feeding 
practice in China today together with the lack of physical activity since small children are held by parents most of the time. No significant associations between children's relative weight (BMI SDS) and eating behaviour were observed in the present study. We also considered if adjusting parental weight could affect the correlation between children's BMI SDS and CEBQ scales. Therefore we also checked the simple correlation without controlling anything. The result remains the same and we therefore suggest that this could probably be explained by the young age of children in this study, as some eating behaviours are harder to detect at early age.

Moreover, different eating behavior in Chinese and European children might also contribute to this negative correlation, because CEBQ was developed on the basis of European children's eating behavior. This was also one main intention to conduct our study.

This study has several limitations. First, the sample population might not be representative for the whole Chinese children at the age of 12-18 months as it was tested only in two cites. Secondly, the parental weight and height were self-reported. In addition, the original CEBQ used oblimin rotation in factor analysis, we used varimax rotation instead, though no difference presented.

Despite of the drawbacks, this investigation pioneered a new field of studying young children's eating behaviours by using CEBQ in a totally different setting (China) and in younger children compared to the original and other studies. Based on our results we suggest certain adjustments in the Chinese version of CEBQ, such as to exclude the questions belonging to EF and SR scales, as they might be irrelevant for such an early age in Chinese children. In addition, our important observations regarding children's gender difference in eating behaviour such as FF and two FRs could have a further study.

\section{Conclusions}

This study validated the CEBQ in the Chinese context and explored Chinese children's eating behaviour and its relationship with obesity in an early age. The Chinese version of CEBQ needs to be adjusted according to the Chinese situation, both because of the younger age, and also due to the different eating behavior in Chinese and European children. However, it offers a great opportunity to study eating behaviour in a different population and compare it with other studies.

\section{Additional material}

Additional file 1: Comparison of the scale structure of this study with Original CEBQ. There are only 19 items determined by this study, however there were 35 items originally developed in CEBQ. In this table, all 35 items are shown, including those items that were not found in this study but were originally developed in CEBQ to compare the structure of original study with this study.

\section{List of abbreviations}

CEBQ: Children's Eating Behaviour Questionnaire; EF: Enjoyment of food; FR: Food responsiveness; EOE: Emotional overeating; DD: Desire to drink; SR: Satiety responsiveness; SE: Slowness in eating; FF: Food fussiness; EFA: Exploratory factor analysis; BMI SDS: Body mass index standard deviation score

\section{Acknowledgements}

We would like to thank all parents and grandparents who completed the questionnaire and for their willingness to communicate about their children' eating behaviours. Great thanks should also be given to National Natural Science Foundation of China (NSFC), and the Special Fund for Basic Scientific Research of Central Colleges, Vinnova, Swedish Council for Working Life and Social Research and the Swedish Order of Freemason. The study was supported by funds from the Swedish Council for Working Life and Social Research (VS, CM, TS), the Jerring Foundation (VS), Karolinska Institutet (VS), the Swedish Order of Freemason (YT C) and VINNOVA (TS). It was also supported by NSFC of China (No. 30800917, JD Zh) and the Special Fund for Basic Scientific Research of Central Colleges, No.2011TS017, JD Zh).

\section{Author details}

'Karolinska Institutet, Department of Clinical Science, Intervention and Technology, Division of Pediatrics, Karolinska University Hospital Huddinge, SE 14186 Stockholm, Sweden. ${ }^{2}$ Tongji Medical College School of Public Health, Huazhong University of Science and Technology, 13 Hangkong RD, Wuhan, 430030, P.R.China.

\section{Authors' contributions}

All authors were involved in all parts of this study, and they all read and approved the final manuscript. The contributions are described below. YT C collected all data, performed the statistical analyses and wrote the whole manuscript. TS was responsible for the design of the study, and supervised the whole process. VS reviewed the manuscript and helped with the statistical analyses. JZh and JD Zh organized data collection in China and reviewed the manuscript. CM has given final approval of the version to be submitted.

\section{Competing interests}

The authors declare that they have no competing interests.

Received: 3 October 2011 Accepted: 24 January 2012

Published: 24 January 2012

\section{References}

1. WHO: Programmes and projects, Global strategy on Diet. Physical activity \& Health, Childhood overweight and obesity. 2010 [http:/www. who.int/dietphysicalactivity/childhood/en/].

2. Ji CY, Cheng TO: Prevalence and geographic distribution of childhood obesity in China in 2005. Int J Cardiol 2008, 131:1-8.

3. $\mathrm{Ji} \mathrm{CY}$, Cheng TO: Epidemic increase in overweight and obesity in Chinese children from 1985 to 2005. Int J Cardiol 2008, 132:1-10.

4. Zhai FY, Wang HJ, Du SF, He Y, Wang ZH, Ge KY, Popkin BM: Prospective study on nutrition transition in China. Nutrition Reviews / 2009, 67(Suppl 1):S56-61.

5. Cheng TO: Fast food, automobiles, television and obesity epidemic in Chinese children. Int J Cardiol 2005, 98:173-174.

6. Schachter S: Obesity and eating. Internal and external cues differentially affect the eating behaviour of obese and normal subjects. Science 1968, 161:751-756.

7. Barkeling B, Ekman S, Rössner S: Eating behaviour in obese and normal weight 11-year-old children. Int J Obes 1992, 16:355-360.

8. Lindgren AC, Barkeling B, Hägg A, Ritzén M, Marcus C, Rössner S: Eating behaviour in Prader Willi Syndrome, normal weight and and obese controls. J Pediatrics 2000, 137(Suppl 1):50-55.

9. Braet C, Van Strien T: Assessment of emotional, externally induced and restrained eating behaviour in nine to twelve-year-old obese and nonobese children. Behav Res Ther 1997, 35:863-873.

10. Archer L, Rosenbaum P, Streiner D: The Children's Eating Behaviour Inventory: reliability and validity results. J Pediatr Psychol 1991, 16:629-642.

11. Wardle J, Guthrie CA, Sanderson S, Rapoport L: Development of the Children's Eating Behaviour Questionnaire. J Child Psychol Psychiatry 2001, 42:963-970. 
12. Carnell S, Wardle J: Measuring behavioural susceptibility to obesity: Validation of the child eating behaviour questionnaire. Appetite 2007, 48(Suppl 1):104-113.

13. Viana V, Sinde S, Saxton JC: Children's eating behaviour questionnaire: associations with BMI in Portuguese children. British Journal of Nutrition 2008, 30:445-450.

14. Sleddens Ester FC, Krmers Stef PJ, Carel Thijs: The association with Body Mass Index in Dutch children aged 6-7. International Uournal of Behavioural Nutrition and Physical Activity 2008, 5:49.

15. Svensson V, Lundborg L, Cao YT, Marcus C, Sobko T: Obesity related eating behaviour patterns in Swedish preschool children and association with age, gender, relative weight and parental weight - factorial validation of the Children's Eating Behaviour Questionnaire. International Journal of Behavioural Nutrition and Physical Activity 2011, 8:134.

16. Jansen A: Overweight children overeat after exposure to food cues. Eating Behaviours 2003, 4(Suppl 2):197-209.

17. Sweetman C, Wardle J, Cooke L: Soft drinks and 'desire to drink' in preschoolers. International Journal of Behavioural Nutrition and Physical Activity 2008, 5(Suppl 1):60.

18. Reau NR, Senturia YD, Lebailly SA, Christoffel KK: Infant and toddler feeding patterns and problems: normative data and a new direction. Pediatric Practice Research Group. J Dev Behav Pediatr 1996, 17:149-153.

19. Carruth BR, Skinner J, Houck K, Moran J, Coletta F, Ott D: The phenomenon of 'picky eater': a behavioural marker in eating patterns of toddlers. J Am Coll Nutr 1998, 17:180-186.

20. Rydell AM, Dahl M, Sundelin C: Characteristics of school children who are choosy eaters. J Genet Psychol 1995, 156:217-229.

21. Wright C, Birks E: Risk factors for failure to thrive: a population-based survey. Child Care Health Dev 2000, 26(Suppl 1):5-16.

22. Serlachius A, Hamer M, Wardle J: Stress and weight change in university students in the United Kingdom. Physiol Behav 2007, 92(4):548-553.

23. Kaplan HI, Kaplan HS: A psychosomatic concept. Am J Psychother 1957, 11(Suppl 1):16-38.

24. Sobko T, Svensson V, Ek A, Ekstedt M, Karlsson H, Johansson E, Cao YT, Hagstromer M, Marcus C: A randomised controlled trial for overweight and obese parents to prevent childhood obesity - Early STOPP (STockholm Obesity Prevention Program). BMC Public Health 2011, 11:336.

25. $\mathrm{Li} \mathrm{H}, \mathrm{Ji} \mathrm{CY}$, Zong XN, Zhang YQ: Body mass index growth curves for Chinese children and adolescents aged 0 to 18 years. Zhonghua Er Ke Za Zhi 2009, 47(Suppl 7):493-498.

26. Ji CY, Report on Childhood Obesity in China (1): Body Mass Idex reference for screening overweight and obesity in Chinese school-age children. Biomedical and environmental sciences 2005, 18:390-400.

27. Rolland-Cachera MF, Sempe M, Guilloud-Bataille M, Patois E, PequignotGuggenbuhl F, Fautrad V: Adiposity indices in children. Am J Clin Nutr 1982, 36:178-184.

28. Bland JM, Altman DG: Cronbach's alpha. British Medical Journal 1997, 314:572.

29. Jiang JX, Roseqvist U, Wang HS, Greiner T, Lian GL, Sarkadi A: Influence of grandparents on eating behaviours of young children in Chinese threegeneration families. Appetite 2007, 48:377-383.

30. Gliem JA, Gliem RR: Calculating, interpreting, and reporting Cronbach's alpha reliability coefficient for Likert-type scales. Midwest research to practice conference in adult, continuing and community education The Ohio state university, Columbus, $\mathrm{OH} ; 2003$.

31. Russell CG, Worsley A: A population-based study of preschoolers' food neophobia and its associations with food preferences. J Nutr Educ Behav 2008, 40:11-19.

32. Dubois L, Farmer A, Girard M, Peterson K, Tatone-Tokuda F: Problem eating behaviours related to social factors and body weight in preschool children: a longitudinal study. Int J Behav Nutr Phys 2007, Act 4:9.

33. Koivisto-Hursti UK, Sjoden P: Food and general neophobia and their relationship with self reported food choice: familial resemblance in Swedish families with children of ages 7-17 years. Appetite 1997, 29:89-103
34. Llewellyn $\mathrm{CH}$, Jaarsveld CHM, Johnson L, Carnell S, Wardle J: Development and factor structure of the Baby Eating Behaviour Questionnaire in the Gemini birth cohort. Appetite 2011, 57:388-396.

35. Addessi E, Galloway AT, Visalberghi E, Birch LL: Specific social influences on the acceptance of novel foods in 2-5-year old children. Appetite 2005, 45:264-271.

doi:10.1186/1479-5868-9-5

Cite this article as: Cao et al:: Eating behaviour patterns in Chinese children aged 12-18 months and association with relative weight factorial validation of the Children's Eating Behaviour Questionnaire. International Journal of Behavioral Nutrition and Physical Activity 2012 9:5.

\section{Submit your next manuscript to BioMed Central and take full advantage of:}

- Convenient online submission

- Thorough peer review

- No space constraints or color figure charges

- Immediate publication on acceptance

- Inclusion in PubMed, CAS, Scopus and Google Scholar

- Research which is freely available for redistribution 